\title{
Role of Multidetector Computed Tomography in Assessment of Maxillofacial Fractures
}

\author{
AWAD A. BESSAR, M.D.*; FARIDA M. AL-FAWAL, M.D.*; DALIA S. ANWAR, M.D.* and \\ IBRAHIM A.M. ALZARGA, M.Sc.** \\ The Department of Radiodiagnosis, Faculty of Medicine, Zagazig University, Egypt* and Sirte University, Libya**
}

\begin{abstract}
Background: Maxillofacial fractures are common and must be radiologically evaluated to detect fractures, to determine their morphology and topography, and to assess adjacent soft-tissue damage.
\end{abstract}

Aim of Study: The aim of the current study was to evaluate the role of MDCT in diagnosis of: Maxillofacial fractures.

Patients and Methods: This prospective cross-sectional study was carried in Radiodiagnosis Department, Zagazig University Hospitals and private center on twenty eight patients complaining from facial trauma with suspected facial fractures referred from Emergency Department, they were 22 males and 6 females, a ages ranged from 17 to 51 years with mean age of 29 years old. Conventional plain radiography and MDCT were performed to all patients.

Results: MDCT examination showed that the most common simple maxillofacial fracture was orbital wall fracture (7 patients), the most common complex fractures zygomaticomaxillary complex fracture (5 patients), the most common orbital bony wall fracture was orbital floor fracture ( 8 patients).

Conclusion: MDCT is the optimal imaging modality for evaluation of maxillofacial fractures, as it can be often visualize complex injuries with a precision unattainable by conventional radiography or clinical examination. Fracture fragments displacement and rotation are easily determined by MDCT and 3D MDCT is the best modality for demonstrating the spatial relationships of fracture fragments in maxillofacial fractures.

Key Words: MDCT-Computed tomography-Maxillofacial fractures.

\section{Introduction}

FRACTURES in the facial region may lead to life threatening situations i.e., airway compromise and profuse blood loss. 2 due to soft tissue swelling, lacerations and pain, it is difficult to physically

Correspondence to: Dr. Ibrahim A.M. Alzarga, E-Mail: ibrahimalzarga@gmail.com. examine the patient with maxillofacial fractures

By appreciating properly the patterns of injury and the implications for clinical management, radiologists can better imply clinically relevant radiology reports and hence facilitating improved communication with referring clinicians. Multidetector Computed Tomography (MDCT) is the imaging modality of choice and is one of the most important imaging tools in evaluation of patients with maxillofacial fractures. It helps in detecting the exact site, number and extent of fractures, displacement of fragments and soft tissue injuries

The added advantage of MDCT is 3D reconstruction and multiplanar reformation in coronal and sagittal planes which are extremely helpful in assessing the bony architecture in large comminuted, displaced and complex fractures involving multiple planes that helps the surgeons for appropriate planning and management. Role of MRI in maxillofacial trauma is to assess the soft tissue injuries, it has excellent soft tissue contrast; and also aids in assessing the patients with neurological deficits. However, it has insignificant role in the evaluation of cortical bone. The role of plain radiographs in assessing maxillofacial trauma have declined as it does not provides adequate information [3].

\section{Patients and Methods}

Twenty eight patients ( 22 male and 6 female) with age mean 39yrs were referred from Emergency Department of Zagazig University Hospitals to Department of Radiodiagnosis, and private center for this prospective cross-sectional study. During 
the period from July 2018 to January 2019 . The study included 28 patients who sustained trauma to the facial skeleton with age range from 17-51 years old. Conventional plain radiography and MDCT were performed to all patients. Informed consent from all participants of the study.

\section{Inclusion criteria:}

- Affected patients with suspicion of maxillofacial fractures and clinically complain.

- Patients with normal X-ray but clinically complain.

\section{Exclusion criteria:}

- Patients with normal findings were excluded from this study.

- Pregnant females.

Methods: All patients were subjected to the following:

Clinical assessment:

1- Full clinical history taking:

A- Personal data include: Patient name, age and sex.

B- Complaint and present history include: History of trauma and associated symptoms.

2- Clinical examination: Patients assessed by the emergency physician then redirected to the Radiology Unit.

\section{Radiodiagnostic imaging:}

1- Conventional radiography: All patients in our study had plain X-ray films on the affected side.

\section{2- MDCT examination:}

-Examination Technique: MDCT examination of the facial region was done for all patients included in this study. All MDCT examinations were carried with a 128-channel MDCT scanner (Philips ingenuity 128) using the following variables: Detector row configuration, $128 \times 1 \mathrm{~mm}$; collimation, $1 \mathrm{~mm}$; slice thickness, $1.25 \mathrm{~mm}$; pitch, 1.375 ; reconstruction interval, $1 \mathrm{~mm} ; 300 \mathrm{mAs} ; 120 \mathrm{kVp}$. To obtain direct axial scans, patients were scanned in supine position with head first towards the gantry without gantry tilt. No specific preparation was required for patients apart of quiet breathing. MDCT protocol consists of volumetric data acquisition commencing below the mandible and ending when frontal sinuses are cleared. Although the direct coronal images were highly delicate than the reformatted images, yet, it is very difficult to get direct coronal images in traumatic maxillofacial fractures. Post processing, the scans were recon- structed and reviewed. Multiplanar Reconstructions (MRP) were obtained using the machine software in coronal and sagittal planes. The thin axial slices MDCT scanner and reconstruction of 3D images which are important adjuncts to axial and MRP images for evaluation of spatial relationships. However, 3D images alone should not be used for the detection and characterization of fractures. Multidetector CT had transformed CT from a trans axial cross-sectional technique to a true 3D imaging modality for arbitrary cut planes as excellent 3D displays of the data volume. Multi-slice CT scanners provide a best results in performance which could be used for reduction of scan time, section collimation, and increase scan length substantially.

-Image interpretation: The axial source images and post-processing images were checked for detecting the presence of facial fractures, the fractures extent and assessment of the soft tissues. Fractures were sub-grouped to nasal fractures, nasoethmoidal fractures, complex fractures of zygomatic malar, fractures of orbital floor, maxillary sinus wall, frontal sinus, jaw, Le Fort and other fractures.

\section{Final diagnosis of the patients:}

Final diagnosis of the patients was reached by operative data during reduction and fixation of the fracture.

\section{Statistical analysis:}

Data were collected, tabulated and analyzed by SPSS 20, software for Windows. The significance level was set at $p<0.05$.

A female patient aged 45 years old admitted to the Emergency Department with after a road traffic accident. MDCT was performed.

\section{Results}

Table (1), showed that the studied group was 22 males (78.6\%) and 6 females (21.4); 19 patients $(67.9 \%)$ were $\leq 30$ years and 9 patients $(32.1 \%)$ were $>30$ the mean age for the studied patients was $29 \pm 10.5$ years. Table (2), showed that road traffic accidents $50 \%$ of patients, fall from height represents $21.4 \%$ of patients, physical assault represents $17.9 \%$ of patients and sports injuries in the represents $10.7 \%$ of the patients.

Table (3), showed that MDCT examination detected simple fractures orbital wall fracture in 7 cases, nasal bone fracture in 4 cases, mandibular fracture 3 cases, maxillary sinus wall fracture 2 cases, fracture maxilla (hard palate and alveolar 
process) 2 cases, isolated zygomatic arch fracture in one case and frontal sinus fracture in one case. Therefore, the most common simple maxillofacial fracture was orbital wall fracture seen in 7 patients. Table (4), showed that MDCT detected complex fractures in the form of zygomatic-maxillary complex fracutre in 5 cases, Le Fort II fracture in 2 cases, Le Fort I fracture in 2 cases, Le Fort III fracture in 2 cases and NOE fracture in one case. Therefore, zygomatico-maxillary complex fracture is the most common complex fractures seen in 5 cases (17.9\%). Table (5), showed that plain X-ray detected simple facial fractures in 17 patients among 28 patients with facial fractures, while MDCT detected simple facial fractures in 20 patients. Table (6), showed that plain X-ray detected 6 complex facial fractures, while MDCT detected 12 complex facial fractures. Table (7), showed that In simple fractures, conventional plain radiography and MDCT had sensitivity of $85 \%$, specificity of $87.5 \%$, positive predictive value of $94.4 \%$ and negative predictive value of $70 \%$. In complex fractures, they had sensitivity of $50 \%$, specificity of $93.8 \%$, positive predictive value of $85.7 \%$ and negative predictive value of $71.4 \%$.

Table (1): Age and gender distribution in 28 patients with maxillofacial fractures.

\begin{tabular}{ll}
\hline Variables & $\mathrm{n}=28$ \\
\hline Age $($ years): & \\
Mean $\pm \mathrm{SD}$ & $29 \pm 10.5$ \\
Range & $17-51$ \\
Age groups: & \\
$\leq 30$ & $19(67.9 \%)$ \\
$>30$ & $9(32.1 \%)$ \\
Gender: & \\
Male & $22(78.6 \%)$ \\
Female & $6(21.4 \%)$ \\
\hline
\end{tabular}

Table (2): Distribution of different maxillofacial fractures in 28 patients according to the MDCT findings.

\begin{tabular}{lll}
\hline & No & $\%$ \\
\hline Orbital fracture & 14 & 50 \\
Nasal fracture & 12 & 42.9 \\
Maxillary sinus wall fracture & 9 & 32.1 \\
Zygomatico-maxillary complex fracture & 5 & 17.9 \\
Mandibular fracture & 5 & 17.9 \\
Fracture maxilla & 4 & 14.3 \\
Frontal sinus fracture & 3 & 10.7 \\
Le Fort II fracture & 2 & 7.1 \\
Le Fort I fracture & 2 & 7.1 \\
Le Fort III fracture & 2 & 7.1 \\
Naso-orbito-ethmoidal fracture & 1 & 3.6 \\
Isolated zygomatic arch fracture & 1 & 3.6 \\
\hline
\end{tabular}

Table (3): Incidence of simple facial fractures in 28 examined patients with maxillofacial fractures.

\begin{tabular}{lcc}
\hline & No & $\%$ \\
\hline Orbital wall fracture & 7 & 25 \\
Nasal fracture & 4 & 14.3 \\
Mandibular fracture & 3 & 10.7 \\
Maxillary sinus wall fracture & 2 & 7.1 \\
Fracture maxilla & 2 & 7.1 \\
Isolated zygomatic arch fracture & 1 & 3.6 \\
Frontal sinus fracture & 1 & 3.6 \\
\hline
\end{tabular}

Table (4): Incidence of complex facial fractures in 28 patients with maxillofacial fractures.

\begin{tabular}{lcc}
\hline & No & $\%$ \\
\hline ZMC & 5 & 17.9 \\
Le Fort II & 2 & 7.1 \\
Le Fort I & 2 & 7.1 \\
Le Fort III & 2 & 7.1 \\
NOE fracture & 1 & 3.6 \\
\hline
\end{tabular}

Table (5): Incidence of different simple facial fractures detected by X-ray and MDCT.

\begin{tabular}{lcc}
\hline & X-ray & MDCT \\
\hline Orbital wall fracture & 5 & 7 \\
Nasal fracture & 4 & 4 \\
Mandibular fracture & 3 & 3 \\
Maxillary sinus wall fracture & 2 & 2 \\
Fracture maxilla & 1 & 2 \\
Frontal sinus fracture & 1 & 1 \\
Isolated zygomatico-arch fracture & 1 & 1 \\
\hline Total & 17 & 20 \\
\hline
\end{tabular}

Table (6): Incidence of different complex facial fractures detected by X-ray and MDCT.

\begin{tabular}{lcc}
\hline & X-ray & MDCT \\
\hline ZMC fracture & 5 & 5 \\
Le Fort II & 0 & 2 \\
Le Fort I & 0 & 2 \\
Le Fort III & 0 & 2 \\
NOE fracture & 1 & 1 \\
\hline Total & 6 & 12 \\
\hline
\end{tabular}

Table (7): Diagnostic performance of X-ray and MDCT in diagnosis of simple and complex fractures.

\begin{tabular}{lcccl}
\hline & Sensitivity & Specificity & PPV & NPV \\
\hline Simple fracture & $85 \%$ & $87.5 \%$ & $94.4 \%$ & $70 \%$ \\
Complex fracture & $50 \%$ & $93.8 \%$ & $85.7 \%$ & $71.4 \%$ \\
\hline
\end{tabular}




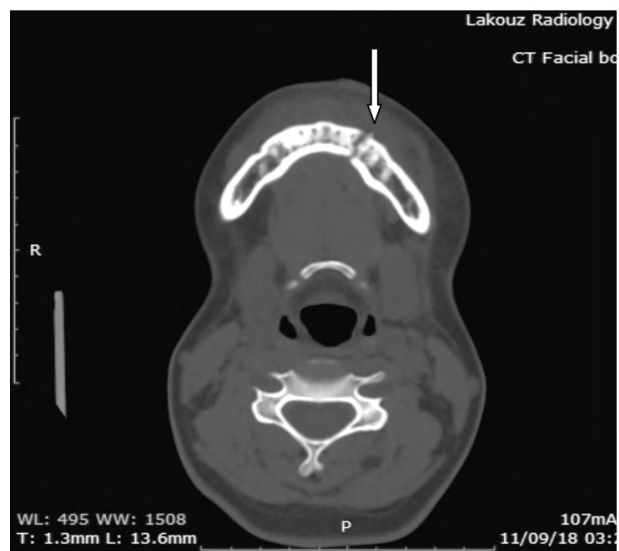

(A)

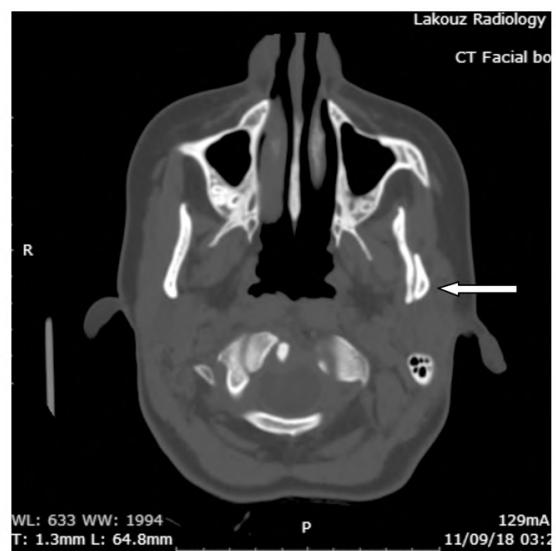

(B)

Fig. (1): A female patient aged 45 years old admitted to the Emergency Department with after a road traffic accident. MDCT was performed. (A, B) Axial MDCT images of facial bones (bone window): Show fracture of the body of the mandible at the LT parasymphyseal line (white arrows). And page (B), show fracture of the left mandibular condylar neck. (White arrows).

Diagnosis: Fractures mandibular body and left mandibular condylar neck.

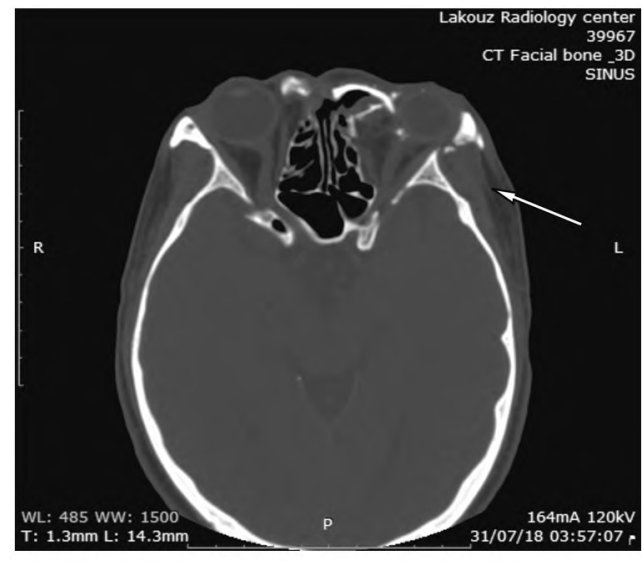

(A)

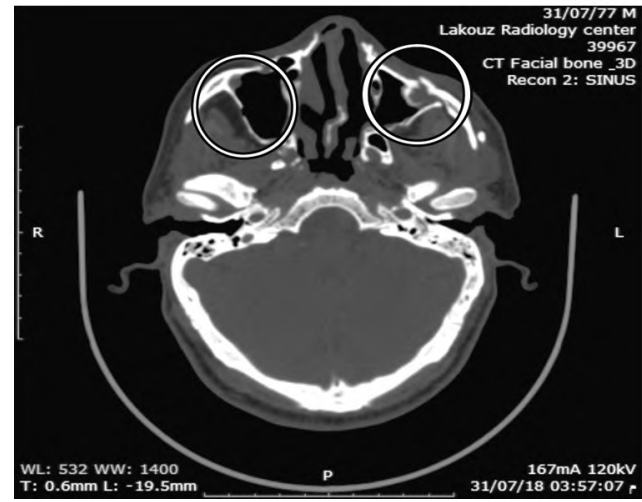

(C)

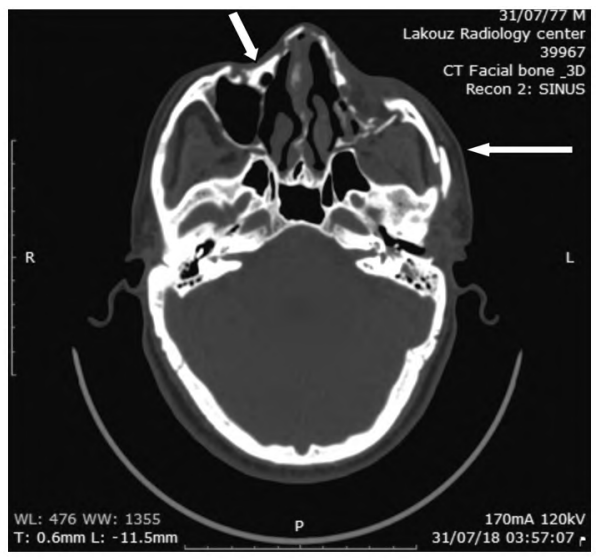

(B)

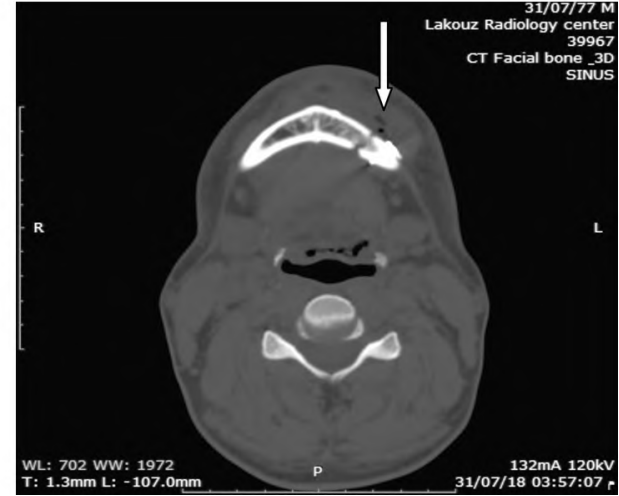

(D)

Fig. (2): A male patient aged 26 years old, admitted to the Emergency Department after falling from the fifth floor during his work as a construction worker. MDCT was performed. (A, B) Axial MDCT of facial bones (bone window) show fracture of both nasal bones left lateral orbital wall, left zygomatic arch (arrows). (C) Axial MDCT image of facial bones (bone window): Shows fracture bilateral maxillary sinus walls (circles). (D) Axial MDCT image of facial bones (bone window): Shows displaced fracture body of mandible (white arrow).

Diagnosis: Left ZMC fractures with right maxillary sinus wall fracture. Bilateral nasal bones and fracture mandible. 


\section{Discussion}

Multislice CT with 3D images provides better perception of the pattern of the fracture lines, and the displacement of the bony fragments thus helping in the faster and improved communication of the information to the referring physician [4].

In our study, majority of the patients were males with number of 22 patients which represents $(78.6 \%)$ of the total, while number of female patients was 6 which represents (21.4\%) of the total. This was in agreement with Ahmad et al., [5] who assessed the role of Multidetector Computed Tomography (MDCT) in patients with facial trauma. They reported that guys speak to $(70 \%)$ of the study and females speak to (30\%) of the study.

In our study, ages ranged from 17 to 51 years and the mean age was 29 years; the most frequent age group was $<30$ years which represents $67.9 \%$ of the total and the least frequent age group was $>30$ years in the form of 9 patients which represents only $32.1 \%$ of the total. Ahmad et al., [5] reported that ages of patients ranged from 12 to 70 years. $55 \%$ of patients were in 1130 years of age and $20 \%$ of patients were in 3140 years of age.

In our study, the common cause of injuries in our study was road traffic accidents in the form of 14 patients which represents $50 \%$ of the total, fall from height in the form of 6 patients which represents $21.4 \%$ of the total, physical assault in the form of 5 patients which represents $17.9 \%$ of the total and sports injuries in the form of 3 patients which represents $10.7 \%$ of the total. These results were in agreement with the study of Downing et al., [6].

Also the study of Ahmad et al., [5] who reported that the most common cause of injury was the road traffic accident $(58.33 \%)$, followed by fall from height (20\%), physical assault (16.66\%) and sport injuries (5\%).

In our study, 28 patients with 60 maxillofacial fractures were examined by MDCT. We found that the most common fracture were orbital fracture seen in 14 cases $(50 \%)$. This was relatively in agreement with Abdel Wahab et al., [7] who reported that orbital cracks were the most successive breaks and were found in 22 cases representing around $73 \%$ and the nasal breaks found in 19 cases representing $63 \%$. But, this was not in agreement with Hwang and You [8] who reported that the orbital divider breaks came third in his exploration representing $(7.6 \%)$ just of the analyzed cases after the nasal and mandibular cracks.
In our study, MDCT examination detected simple fractures in the form of: Orbital wall fracture in 7 cases, nasal bone fracture seen in 4 cases, mandibular fracture seen in 3 cases, maxillary sinus wall fracture seen in 2 cases, fracture maxilla (hard palate and alveolar proceess) in 2 cases, isolated zygomatic arch fracture in one case and frontal sinus fracture in one case. Therefore, the most common simple maxillofacial fracture was orbital wall fracture seen in 7 patients.

MDCT detected complex fractures in the form of zygomatic-maxillary complex fracutre in 5 cases, Le Fort II fracture in 2 cases, Le Fort I fracture in 2 cases, Le Fort III fracture in 2 cases and NOE fracture in one case. Therefore, zygomaticomaxillary complex fracture is the most common complex fractures seen in 5 cases $(17.9 \%)$.

In our study, MDCT detected orbital bony wall fractures in 14 patients. MDCT detected orbital floor fractures in 8 patients accounting for about $57.1 \%$ of orbital fractures, lateral orbital wall fracture in 6 patients $(42.86 \%)$, medial orbital wall fracture in 4 patient $(28.6 \%)$ and orbital roof fracture in 3 patients accounting for about $21.4 \%$. Therefore, orbital floor fracture is the most common orbital bony wall fracture seen in 8 cases $(57.1 \%)$. This was in agreement with Ahmad et al., [5] who found that the fractures of orbital floor were the most common fracture accounting for $36 \%$. From the orbital floor fractures there were 21 patients 7 $(33.33 \%)$ had blow out fracture of the orbit while $3(14.28 \%)$ patients had associated herniation of inferior rectus muscle and orbital fat in the maxillary sinus.

Abdel Wahab et al., [7] reported that frequency of orbital divider cracks as per the site spoke to as: Average divider $63.6 \%$, horizontal divider $59 \%$, story $41 \%$ and rooftop $31.8 \%$ of cases. Nasal fracture was seen in 19 cases accounting for about $63 \%$ and maxillary bone fracture was detected in 17 cases accounting for about $56 \%$.

In our study, MDCT detected fracture mandible in 5 cases. MDCT detected fracture of mandibular body in 2 patients. Fracture of mandibular symphysis was detected in 2 patients. Fracture of the condylar process was detected in one patient. Also, MDCT detected coronoid process in one patient. The distribution of mandibular fractures according to the fracture site.

This was relatively in agreement with Ozakaya et al., [9] who reported that $30 \%$ had isolated mandibular fracture, while $10 \%$ had mandibular fracture associated with other maxillofacial frac- 
tures. Also, Eskitascioglu et al., [10] reported that the most widely recognized site of mandibular crack was the body spoke to by $52.2 \%$ and the slightest regular site was the coronoid process spoke to by $0.2 \%$.

In our study, plain X-ray detected simple facial fractures in 17 patients and 6 complex facial fractures among 28 patients with facial fractures, while MDCT detected simple facial fractures in 20 patients and 12 complex facial fractures in 8 patients.

Mithani et al., [11] reported that $50 \%$ of the patients with maxillofacial trauma had intracranial injuries. Motamedi et al., [12] reported that Le Fort II fracture was the most common among the Le Fort fractures with a percent of $7.6 \%$.

Abdel Wahab et al., [7] reported that the largest fractures included the zygomatico-maxilla accounting about $(27 \%)$. Naso-orbito-ethmoidal fractures were detected in 6 patients accounting for $20 \%$. Reformatted coronal and sagittal CT pictures are of high caliber and have been appeared to be solid and precise in surgical reconstructive endeavors. For each kind of complex cracks, there was a particular sweep that was the hint of the case. Axial segments were the best to analyze the back mass of the maxillary antrum, the pterygoid plates, the taste hard sense, dentoalveolar fragments, the zygomatic curve and body, and the horizontal mass of the circle. Coronal areas recorded the best pictures to dissecting the foremost mass of the maxilla, the mediocre orbital edge, the sense of taste, and the orbital floor. Likewise, the average and posterohorizontal dividers of the maxillary sinus were satisfactorily surveyed by the coronal images.

In our study, we found that in simple fractures, conventional plain radiography and MDCT had a sensitivity of $85 \%$, specificity of $87.5 \%$, positive predictive value of $94.4 \%$ and negative predictive value of $70 \%$. In complex fractures, they had a $50 \%$ sensitivity, $93.8 \%$ specificity, $85.7 \%$ positive predictive value and $71.4 \%$. Negative predictive value which in agreement with the study of Schuknecht and Graetz [13] who reported that the sensitivity of Multidetector Computed Tomography (MDCT) to detect simple maxillofacial fractures is $100 \%$, whereas conventional X-rays had only $86 \%$ sensitivity.

Ahmad et al., [5] concluded that the injuries in maxillofacial region was the most common emergency requiring early and accurate diagnosis for proper management. MDCT has high sensitivity and specificity and has high accuracy for detection of fracture.
Raju et al., [14] identified and classified maxillofacial fractures using multi-slice Computed Tomography (CT) and identified the advantages of three-dimensional (3D) images over twodimensional axial images in evaluation of maxillofacial injuries. They demonstrated that MDCT provides excellent spatial resolution in the evaluation of fractures in the maxillofacial region. 3D rendered images provide a better aspect of the pattern of the fracture lines and the the bony fragments displacement, especially in the mandible and zygomatic bone which could be help in the faster and improved communication of the information to the specialist surgeon. 3D images were also better in the identification of Le Fort fracture lines. MDCT is an accurate, noninvasive technique for the evaluation of patients with maxillofacial injuries with an added advantage of shorter scan time and easy availability.

So, we found in our study that multidetector CT is the examination of decision and is the methodology frequently utilized for imaging assessment as a part of patients of maxillofacial injury. It gives better outline of rigid and delicate tissue highlights, offers both multiplanar and three-dimensional picture remaking, and can be performed more rapidly than radiography, with less demanding patient situating. Precise depiction of facial cracks and intricacies is of fundamental significance for surgical arranging and suitable administration.

Facial injuries were commonly encountered emergencies which required early diagnosis and management. Road traffic accidents and social violence were the common reasons which could lead to increase in the frequency of maxillofacial injuries. The complex anatomy of the facial bones require multiplanar imaging techniques for a detailed evaluation. The main purpose of diagnostic imaging was to detect and localize the number and site of facial fractures in addition to soft tissue injuries.

\section{Conclusion:}

MDCT is the optimal imaging modality for detection of facial skeletal fractures, as it can be often visualize complex injuries with a precision unattainable by conventional radiography or clinical examination. It offers excellent spatial resolution, which in turn enables exquisite multiplanar reformations, and 3D reconstructions, allowing enhanced diagnostic accuracy and road map for surgical planning. Fracture fragments displacement and rotation are easily determined by MDCT and 3D MDCT is the best modality for demonstrating the spatial relationships of fracture fragments in com- 
plex facial injuries. So after stabilization of the traumatized patient, MDCT is often the first and the most important imaging procedure a polytraumatized patient will need.

\section{References}

1- GOH S.H. and LOW B.Y.: Radiologic screening for midfacial fractures: A single 30 degree occipitomental view is enough. J. Trauma, 52: 688-92, 2002.

2- SALVOLINI U.: Traumatic injuries: Imaging of facial injuries. Eur. Radiol., 12: 1253-61, 2002.

3- SOM P.M. and BRANDWEIN M.S.: Facial fractures and post-operative findings. En: Som PM, Curtin HD (eds). Head and neck imaging. Mosby, St. Louis, 374-438, 2002.

4- RAJU N.S., ISHWAR P. and BANERJEE R.: Role of multislice computed tomography and three-dimensional rendering in the evaluation of maxillofacial injuries. Journal of Oral and Maxillofacial Radiology, 5 (3): 67, 2017.

5- AGARWAL A., KUMAR N. and SHARMA N.: 2D and 3D CT scan-A diagnostic adjunct or necessity in maxillofacial trauma. Indian Journal of Dentistry, 3 (4): 1859, 2012.

6- AHMAD K., RAUNIYAR R.K., KUMAR M.G., et al.: Multidetector computed tomographic evaluation of maxillofacial trauma. Asian Journal of Medical Sciences, 5 (4): 39-44, 2014.

7- DOWNING A., COTTERILL S. and WILSON R.: The epidemiology of assault across the West Midlands. J. Emerg. Med., 20: 434-7, 2003.

8- ABDEL WAHAB M.A., IBRAHEIM M.A. and OSMAN
N.M.: The role of multi detector computerized tomography in evaluation of maxillofacial fractures. The Egyptian Journal of Radiology and Nuclear MedicineHYPERLINK "http://www.sciencedirect.com/science/journal/0378603X/ 45/1", 45 (1): 97-104, 2014.

9- HWANG K. and YOU S.H.: Analysis of facial bone fractures: An 11-year study of 2094 patients. Indian J. Plast. Surg., 43 (1): 42-8, 2010.

10- OZAKAYA O., TURGUT G. and KAYAKLI M.U.: A retrospective study on the epidemiology and treatment of maxillofacial fractures. Ulus Travma Acil Cerrahi Dreg., 15 (3): 262-6, 2009.

11- ESKITASCIOGLU T., OZAZGAN I., CORUH A., et al.: Fractures of the mandible: A 20-year retrospective analysis of 753 patients, Turkish Journal of Trauma \& Emergency Surgery, 19 (4): 348-56, 2014.

12- MITHANI S.K., STHILAIRE H., BROOKE B.S., et al.: Predictable patterns of intracranial and cervical spine injury in craniomaxillofacial trauma: Analysis of 4786 patients. Plast. Reconstr. Surg., 123: 1293-301, 2009.

13- MOTAMEDI M.H., DADGAR E. and EBRAHIMI A.: Curbing Road Traffic Accidents-The Major Cause of Facial Fractures. International Journal of Emergency Mental Health and Human Resilience, 16 (69-70): 15224821, 2014.

14- SCHUKNECHT B. and GRAETZ K.: Radiologic assessment of maxillofacial, mandibular and skull base trauma. Eur. Radiol., 15 (3): 560-8, 2005.

15- RAJU N.S., ISHWAR P. and BANERJEE R.: Role of multislice computed tomography and three-dimensional rendering in the evaluation of maxillofacial injuries. J. Oral Maxillofac Radiol., 5: 67-73, 2017.

\section{دور الأثمة المقمعيلة متعلددة المقاطع فى تقييه كسور الوجه والفكين منهين}

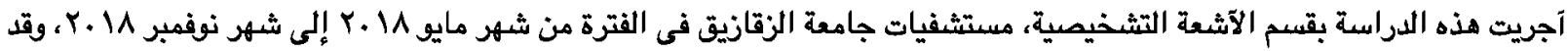

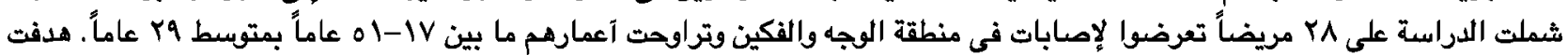

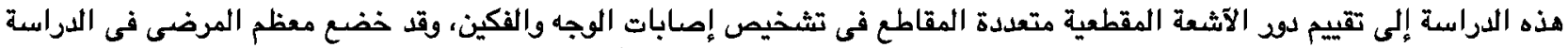

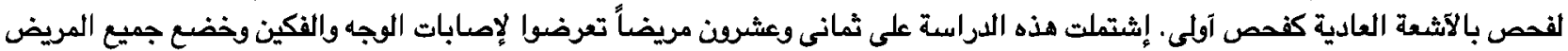

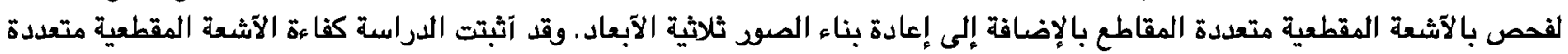

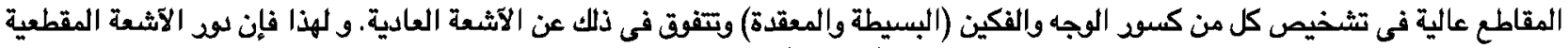

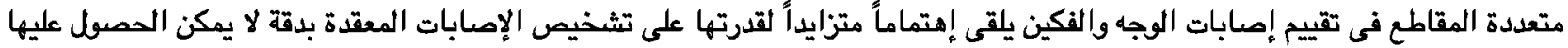

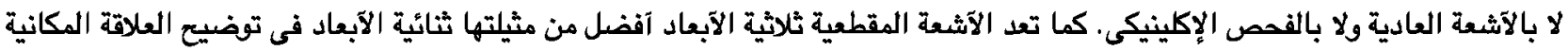

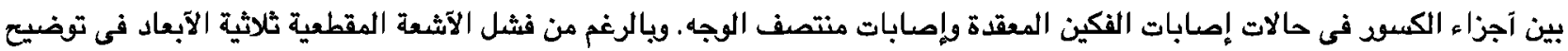
إصابات الآنسجة الرخوة، إلا آنها ساعدات الجراحين الفين الهقدة التخطيط المسبق اللتدخل الجراحى.
} 